\title{
Analytic Solution for Aerodynamic Noise Generated by Plates with Spanwise-Varying Trailing Edges
}

\author{
LORNA J. AYTON † \\ Department of Applied Mathematics and Theoretical Physics, University of \\ Cambridge, Wilberforce Road, CB3 0WA, UK
}

(Received 18 May 2018)

This paper presents an analytic solution for aerodynamic noise generated by an unsteady wall pressure gust interacting with a spanwise variable trailing edge in a background steady uniform flow. Viscous and non-linear effects are neglected. The WienerHopf method is used in conjunction with a non-orthogonal coordinate transformation and separation of variables to permit analytical progress. The solution is obtained in terms of a tailored modal expansion in the spanwise coordinate, however only finitely many modes are cuton, therefore the far-field noise can be quickly evaluated. The solution gives insight into the potential mechanisms behind the reduction of noise for plates with serrated trailing edges compared to those with straight edges. The two mechanisms behind the noise reduction are an increased destructive interference in the far field, and a redistribution of acoustic energy from low cuton modes to higher cutoff modes. Five different test case trailing-edge geometries are considered. The analytic solution identifies which geometries are most effective in different frequency ranges; geometries which promote destructive interference are best at low frequencies, whilst geometries which promote a redistribution of energy are better at high frequencies.

\section{Introduction}

Trailing-edge noise, the noise generated by a turbulent boundary layer scattering off the sharp trailing edge of a rigid structure, is a fundamental source of structural noise for aircrafts and wind turbines. The environmental impact and local disturbance of take-off noise is of particular concern; the Advisory Council for Aerospace Research in Europe (ACARE) set targets in 2001 to reduce perceived noise levels by $50 \%$ by 2020 , which have been extended in the new Flightpath 2050 vision to a reduction of $65 \%$ (relative to noise levels in 2000) by 2050 (European Commission 2011). Similarly, increasing demands on sustainable energy require more wind farms to be constructed, but local residents' complaints about noise often block these plans, hence designs capable of reducing this noise are required. Currently many wind turbines are braked to control noise levels, leading to up to a $25 \%$ power reduction; new noise reduction technologies could improve power output without increasing noise levels.

A great deal of recent work on trailing-edge noise comprises of novel design ideas aimed at reducing trailing-edge noise. These new designs, typically inspired by the silent flight of owls (Graham 1934), are widely varied. Such designs include; serrated trailing edges (Lyu et al. 2016; Oerlemans 2016; Chong \& Vathylakis 2015; Huang 2017), porous and flexible trailing edges (Schlanderer \& Sandberg 2016; Cavalieri et al. 2016; Jaworski \& 
Peake 2013; Herr 2006), trailing-edge finlets (Clark et al. 2016), active boundary layer flow control (Wolf et al. 2014), rough surfaces near the trailing edge (Clark et al. 2014). Whilst trailing-edge serrations seem to be a simple adaptation, there are hugely varying design ideas, from a simple sawtooth serration (Lyu et al. 2016), to a sawtooth serration which varies along the span of the blade (Koegler et al. 2009), to serrations comprising of stiffening ribs (Oerlemans \& Olsen 2014), and novel iron-shaped serrations (Avallone et al. 2017). In part this is due to a lack of full understanding of the mechanisms behind the noise reduction, hence no optimal design exists. It is this adaptation, the serration, which we focus on in the paper.

Predominantly the work on noise reduction through the use of serrated trailing edges is experimental (Moreau \& Doolan 2013; Oerlemans 2016; Chong et al. 2013; Chong \& Vathylakis 2015) or numerical (van der Velden et al. 2017; Jones \& Sandberg 2010, 2012; Karimi et al. 2017; Sanjose et al. 2014). With only these approaches it is very difficult to ascertain noise-reduction mechanisms. Additionally, within the experimental results, there is a large variation in the level of noise predicted from serrated trailing edges dependent on aerofoil geometry, angle of attack, and how the serrations are fitted to the baseline aerofoil (Dassen et al. 1996; Leon et al. 2017). Theoretical approaches should allow for a greater understanding of the relationships between spanwise plate geometry at the trailing edge and the overall levels of far-field noise through analytic expressions of the far-field noise. Despite early theoretical work by Howe (1998) for the noise generated by a wall pressure spectrum scattering off a sinusoidal (Howe 1991a) or sawtooth (Howe 1991b) trailing edge, it is known this approach greatly over predicts the potential noise reductions (Lyu et al. 2016; Winkler et al. 2010), hence greater analytical investigation is required. The approach used by Howe is one using a Green's function, and since these results are known to be inaccurate, different analytic methods should be sought.

Other early theoretical approaches to straight-edge interaction problems have used the Wiener-Hopf method, such as the Sommerfeld diffraction problem (Crighton et al. 1996), or Schwartzchild technique, used by Amiet (1976) in perhaps the most famous result for unsteady disturbances convected past a (straight) trailing edge. Recent theoretical approaches to serrated edges, Lyu et al. (2016) and Huang (2017), still use Schwartzchild and Wiener-Hopf approaches respectively, however due to the spanwise dependency of the edge, both use a Fourier series to enable analytic progress. Unfortunately, by decomposing the problem as a Fourier series, both Lyu et al. (2016) and Huang (2017) obtain equations which cannot be solved exactly; Lyu et al. (2016) implements an iterative procedure, whilst Huang (2017) must use a pole-removal technique (Noble 1958) to factorise an infinite matrix. Both final semi-analytic solutions also involve infinite sums due to the Fourier series. As the solutions are not given in closed analytical forms it is still difficult from these to determine the true mechanisms for noise reduction.

This paper will therefore present a new analytical approach, based on the Wiener-Hopf technique, for trailing-edge noise generated by a flat plate with a serrated trailing edge. We do so by adapting the approach used in Ayton \& Kim (2018) for leading-edge noise from a serrated plate, to a trailing-edge problem. This approach does not use a Fourier series expansion, instead via a non-orthogonal change of coordinates seeks a separable solution in the spanwise and normal coordinates. The benefit of this is modes are not imposed on the solution, but are solved for as a result of the governing equations. The approach was first used by Envia (1988) who considered a swept edge in a finite-span channel.

In addition, due to the great variation in serrated trailing-edge designs, we will allow for an arbitrary spanwise trailing-edge geometry, allowing investigation into an optimal geometry and a greater understanding of how different geometries can affect the 


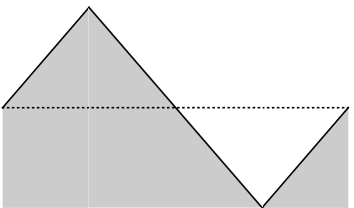

(a) Sawtooth

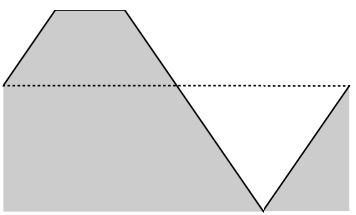

(d) Chopped peak
Trailing-Edge Serrations

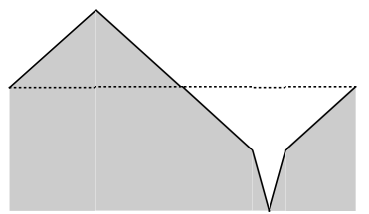

(b) Slitted v-root

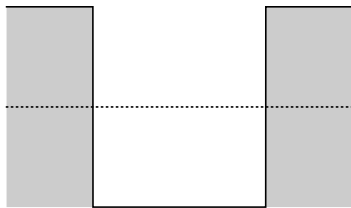

(e) Square wave

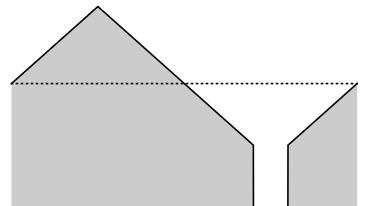

(c) Slitted u-root

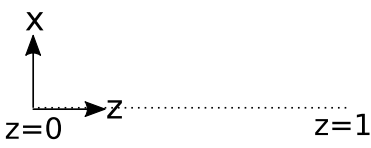

(f) Coordinate system

Figure 1: Trailing-edge geometries. The shaded area denotes the rigid plate, with border $x=\tilde{c} F(z)$, and the dotted line in each figure denotes $x=0$.

total far-field noise. For simplicity we focus on piecewise linear periodic trailing-edges although any periodic smooth (single-valued) trailing edge would also be approachable via this technique. The mechanisms for noise reduction will be inferred from the analytic solutions.

The layout of this paper is as follows. In Section 2 we formulate the mathematical model for a trailing-edge gust scattering off a serrated edge, with piecewise linear periodic geometry. In Section 3 we obtain an analytic solution to the governing equations by using the Wiener-Hopf method. Section 4 contains far-field noise results for five specific test case geometries, and uses the solutions to infer noise-reduction mechanisms. Finally we present conclusions in Section 5 .

\section{Formulation of the Mathematical Problem}

We consider the interaction of an unsteady wall pressure gust convected at velocity $U_{c}$ in uniform flow of Mach number $M$ over a semi-infinite flat plate with a spanwise periodic trailing edge. The semi-infinite plate assumption (which is ideal for applying the Wiener-Hopf technique) will be accurate at mid- and high-range frequencies since any leading-edge rescattering (also known as backscattering) in these cases will be small (Roger \& Moreau 2005, 2009). To simplify the problem we consider a single wavelength of the trailing-edge geometry, and non-dimensionalise lengths by this wavelength, thus restrict to the spanwise region $0 \leqslant z \leqslant 1$. We impose periodic boundary conditions across $z=0,1$. The blade lies in region $x<\tilde{c} F(z), y=0$, with $x$ denoting the streamwise direction, and $\tilde{c}$ permitting a variable 'tip-to-root' ratio, i.e. a varying difference of the height of the trailing-edge geometry. We non-dimensionalise velocities by the far upstream steady velocity, $U=M c_{0}$, where $c_{0}$ is the speed of sound.

We restrict the trailing-edge geometry such that $F(z)$ is a piecewise linear function, and we specifically focus on five different test cases; sawtooth serration, slitted v-root serration, slitted u-root serration, chopped peak serration, and square wave. Our test case geometries are depicted in Figure 1, although the results are applicable to any piecewise linear or smooth, single-valued geometry. Note in all geometry cases, the $z=0,1$ boundaries of the periodic function are chosen to be away from any region of discontinuity of the edge. This ensures the solution captures any influence of the discontinuities. 
In accordance with the model by Lyu et al. (2016), the wall pressure gust has prescribed pressure

$$
p_{i}=P_{i} \mathrm{e}^{\mathrm{i}\left(k_{1} x+k_{3} z-\omega t\right)},
$$

where $P_{i}$ is the magnitude of the incident pressure, and $k_{1,3}$ are the wavenumbers in the streamwise and spanwise directions respectively. For simplicity, since the scattered solution depends linearly on the magnitude of the incident field, we set $P_{i}=1$.

The scattered pressure, $p \mathrm{e}^{-\mathrm{i} \omega t}$, satisfies the compressible convective Helmholtz equation,

$$
\beta^{2} \frac{\partial^{2} p}{\partial x^{2}}+\frac{\partial^{2} p}{\partial y^{2}}+\frac{\partial^{2} p}{\partial z^{2}}+2 \mathrm{i} k M \frac{\partial p}{\partial x}+k^{2} p=0,
$$

subject to boundary conditions of zero normal velocity on the rigid surface

$$
\left.\frac{\partial p}{\partial y}\right|_{y=0}=0 \quad x<\tilde{c} F(z)
$$

and zero pressure jump across the wake downstream of the plate

$$
\left.\Delta p\right|_{y=0}=-\mathrm{e}^{\mathrm{i}\left(k_{1} x+k_{3} z\right)} \quad x>\tilde{c} F(z),
$$

where $\Delta$ denotes the jump in value across $y=0_{+}$and $y=0_{-}$. Here $\beta^{2}=1-M^{2}$ and $k=\omega / c_{0}$. We suppose the wake consists of a flat vortex sheet along $y=0, x>\tilde{c} F(z)$.

We finally impose a spanwise periodicity condition for the solution;

$$
\left.p\right|_{z=1}=\left.p\right|_{z=0} \mathrm{e}^{\mathrm{i} k_{3}},\left.\quad \frac{\partial p}{\partial z}\right|_{z=1}=\left.\frac{\partial p}{\partial z}\right|_{z=0} \mathrm{e}^{\mathrm{i} k_{3}} .
$$

To simplify the governing equation following Roger et al. (2013) we use a convective transform

This results in

$$
h=p(x, y, z) \mathrm{e}^{\mathrm{i} k M x / \beta^{2}} .
$$

$$
\begin{gathered}
\beta^{2} \frac{\partial^{2} h}{\partial x^{2}}+\frac{\partial^{2} h}{\partial y^{2}}+\frac{\partial^{2} h}{\partial z^{2}}+\left(\frac{k}{\beta}\right)^{2} h=0, \\
\left.\frac{\partial h}{\partial y}\right|_{y=0}=0 \quad x<\tilde{c} F(z), \\
\left.\Delta h\right|_{y=0}=-\mathrm{e}^{\mathrm{i}\left(\tilde{k}_{1} x+k_{3} z\right)} \quad x>\tilde{c} F(z) . \\
\left.h\right|_{z=1}=\left.h\right|_{z=0} \mathrm{e}^{\mathrm{i} k_{3}},\left.\quad \frac{\partial h}{\partial z}\right|_{z=1}=\left.\frac{\partial h}{\partial z}\right|_{z=0} \mathrm{e}^{\mathrm{i} k_{3}},
\end{gathered}
$$

where $\tilde{k_{1}}=k_{1}+k M \beta^{-2}$.

We now use a non-orthogonal change of coordinates as done so by Roger et al. (2013) to make the boundary conditions more amenable to the Wiener-Hopf technique by removing the $z$ dependency of the half-regions on which the boundary conditions are prescribed (i.e rather than have $x \gtrless \tilde{c} F(z)$, have some $\xi \gtrless 0$ ).

We choose the following variables

$$
\begin{aligned}
\xi & =\frac{x}{\beta}-c F(z), \\
\eta & =y, \\
\zeta & =z,
\end{aligned}
$$


where $c=\tilde{c} / \beta$. This converts the governing equation and boundary conditions, (2.7) to

$$
\begin{aligned}
\left(1+c^{2} F^{\prime}(\zeta)^{2}\right) \frac{\partial^{2} h}{\partial \xi^{2}}+\frac{\partial^{2} h}{\partial \eta^{2}}+\frac{\partial^{2} h}{\partial \zeta^{2}} & -2 c F^{\prime}(\zeta) \frac{\partial^{2} h}{\partial \xi \partial \zeta}-c F^{\prime \prime}(\zeta) \frac{\partial h}{\partial \xi}+\left(\frac{k}{\beta}\right)^{2} h=0 \\
\left.\frac{\partial h}{\partial y}\right|_{\eta=0} & =0 \quad \xi<0, \\
\left.\Delta h\right|_{\eta=0} & =-\mathrm{e}^{\mathrm{i} \delta \xi+\mathrm{i} \tilde{k}_{1} c F(\zeta)+\mathrm{i} k_{3} \zeta \quad \xi>0} \\
\left.h\right|_{\zeta=1} & =\left.h\right|_{\zeta=0} \mathrm{e}^{\mathrm{i} k_{3}}, \\
\left.\frac{\partial h}{\partial \zeta}\right|_{\zeta=1} & =\left.\frac{\partial h}{\partial \zeta}\right|_{\zeta=0} \mathrm{e}^{\mathrm{i} k_{3}},
\end{aligned}
$$

where $\delta=\tilde{k}_{1} \beta$.

This completes the formulation of the mathematical model.

\section{Analytic solution}

We proceed to solve (2.9) by seeking a separable solution in the $\eta$ and $\zeta$ coordinates. We note that in Roger et al. (2013) the authors correctly identify that the physical space governing equation, (2.9), cannot be separated as the coefficients of the $\xi$ derivatives depend on $\zeta$. However following Ayton \& Kim (2018) we see that in Fourier space (transforming the $\xi$ variable), the equation becomes separable.

We therefore first apply a Fourier transform in the $\xi$ variable,

$$
H(\lambda, \eta, \zeta)=\int_{-\infty}^{\infty} h(\xi, \eta, \zeta) \mathrm{e}^{\mathrm{i} \lambda \xi} d \xi
$$

then separate the solution into $\eta$ and $\zeta$ dependencies, $H(\lambda, \eta, \zeta)=Y(\lambda, \eta) Z(\lambda, \zeta)$, with separation constant $\chi$. This results in equations

$$
Y^{\prime \prime}+\left(\left(\frac{k}{\beta}\right)^{2}-\lambda^{2}-\chi^{2}\right) Y=0
$$

and

$$
Z^{\prime \prime}+2 \mathrm{i} \lambda c F^{\prime} Z^{\prime}+\left(\mathrm{i} \lambda c F^{\prime \prime}-\lambda^{2} c^{2}\left(F^{\prime}\right)^{2}+\chi^{2}\right) Z=0 .
$$

Eq (3.2) has solutions

$$
Y(\lambda, \eta)=\operatorname{sgn}(\eta) \mathrm{e}^{-|\eta| \sqrt{\lambda^{2}-w^{2}}}
$$

where

$$
w^{2}=\left(\frac{k}{\beta}\right)^{2}-\chi^{2},
$$

and we have enforced a radiation condition as $y \rightarrow \infty$.

Eq (3.3) has solutions

$$
Z(\lambda, \zeta)=\mathrm{e}^{-\mathrm{i} \lambda c F(\zeta)}(A(\lambda) \cos (\chi \zeta)+B(\lambda) \sin (\chi \zeta)) .
$$

We impose the periodicity boundary conditions to $Z$ to solve for $\chi$ and eliminate one of $A, B$. This yields allowable modes;

$$
Z_{n}(\lambda, \zeta)=\mathrm{e}^{-\mathrm{i} \lambda c F(\zeta)} \mathrm{e}^{-\mathrm{i} k_{3} \zeta+2 n \pi \mathrm{i} \zeta},
$$


and a general solution given by

$$
H(\lambda, \eta, \zeta)=\sum_{n=-\infty}^{\infty} A_{n}(\lambda) \operatorname{sgn}(\eta) \mathrm{e}^{-|\eta| \sqrt{\lambda^{2}-w_{n}^{2}}} Z_{n}(\lambda, \zeta),
$$

where

$$
w_{n}^{2}=\left(\frac{k}{\beta}\right)^{2}-\chi_{n}^{2}, \quad \chi_{n}= \pm k_{3}+2 n \pi .
$$

Importantly, rather than applying a Fourier series to deal with the $\zeta$ variable, we have allowed for any permitted separable solution. This has shown specific spanwise modes are a direct result of the governing equation.

Now we have an expression for the Fourier transform of the solution, (3.8), we can use the $\eta$ boundary conditions, $(2.9 b)$ and $(2.9 c)$, and apply the Wiener-Hopf technique to solve for $A_{n}(\lambda)$.

First we transform $(2.9 b)$ to obtain

$$
-\sum_{n=-\infty}^{\infty} A_{n}(\lambda) \sqrt{\lambda^{2}-w_{n}^{2}} Z_{n}(\lambda, \zeta)=U_{+}(\lambda, \zeta)
$$

where a subscript + denotes a function that is analytic in the upper half $\lambda$-plane. Aside from its region of analyticity, the function $U_{+}$is formally unknown.

Second we transform $(2.9 c)$ to obtain

$$
2 \sum_{n=-\infty}^{\infty} A_{n}(\lambda) Z_{n}(\lambda, \zeta)=U_{-}(\lambda, \zeta)+\left(\frac{\mathrm{e}^{\mathrm{i} \tilde{k}_{1} c F(\zeta)+\mathrm{i} k_{3} \zeta}}{\mathrm{i}(\lambda+\delta)}\right)_{+},
$$

where a subscript - denotes a function that is analytic in the lower half $\lambda$-plane. Aside from its region of analyticity, the function $U_{-}$is formally unknown.

Since the $Z_{n}$ are a complete orthogonal basis we can expand the right hand sides of (3.10) and (3.11) in terms of this basis. Furthermore the $Z_{n}$ are entire functions of $\lambda$ thus do not affect the domains of analyticity of any functions. We therefore write

$$
\begin{array}{r}
U_{ \pm}(\lambda, \zeta)=\sum_{n=-\infty}^{\infty} U_{ \pm}^{n}(\lambda) Z_{n}(\lambda, \zeta), \\
\left(\frac{\mathrm{e}^{\mathrm{i} \tilde{k}_{1} c F(\zeta)+\mathrm{i} k_{3} \zeta}}{\mathrm{i}(\lambda+\delta)}\right)_{+}=\left(\frac{1}{\mathrm{i}(\lambda+\delta)}\right)_{+} \sum_{n=-\infty}^{\infty} E_{n}(\lambda) Z_{n}(\lambda, \zeta) .
\end{array}
$$

The $U_{ \pm}^{n}$ are unknown functions which are analytic in the upper/lower half plane, whereas the $E_{n}$ are known entire functions that can be determined via

$$
E_{n}(\lambda)=\int_{0}^{1} \mathrm{e}^{i \tilde{k}_{1} c F(\zeta)+\mathrm{i} k_{3} \zeta} \bar{Z}_{n}(\bar{\lambda}, \zeta) d \zeta .
$$

We use orthogonality of the $Z_{n}$ to consider each mode separately, and combine (3.10) and (3.11) to form a single Wiener-Hopf equation for the $n$th mode;

$$
2\left(A_{n}(\lambda) \sqrt{\lambda-w_{n}}\right)_{+}=\left(U_{-}^{n}(\lambda) \sqrt{\lambda-w_{n}}\right)_{-}+\frac{E_{n}(\lambda)\left(\sqrt{\lambda-w_{n}}\right)_{-}}{\mathrm{i}(\lambda+\delta)_{+}} .
$$

We additively factorise the final term on the right hand side to give

$2\left(A_{n}(\lambda) \sqrt{\lambda-w_{n}}\right)_{+}=\left(U_{-}^{n}(\lambda) \sqrt{\lambda-w_{n}}\right)_{-}$ 


$$
+E_{n}(\lambda)\left(\left[\frac{\left(\sqrt{\lambda-w_{n}}\right)_{-}}{\mathrm{i}(\lambda+\delta)_{+}}-\frac{\left(\sqrt{-\delta-w_{n}}\right)_{-}}{\mathrm{i}(\lambda+\delta)_{+}}\right]_{-}+\left[\frac{\left(\sqrt{-\delta-w_{n}}\right)_{-}}{\mathrm{i}(\lambda+\delta)_{+}}\right]_{+}\right),
$$

which is decomposed into functions which are analytic in the upper and lower half planes. To complete the Wiener-Hopf method, we must apply Liouville's theorem to determine an entire function which both + and - contributions to (3.15) equal (by analytic continuation).

To enable the use of Liouville's theorem in the Wiener-Hopf method we must consider the large $\lambda$ behaviour of each term in (3.14). Large $\lambda$ corresponds to small $x$ through inverting the Fourier transform, therefore we consider the physical possibilities for $A_{n}(\lambda) \sqrt{\lambda^{2}-w_{n}^{2}}$ and $U_{-}^{n}(\lambda)$, which are modal coefficients of the scattered normal velocity just above the wake $(x>0)$, and the scattered pressure jump across the surface of the plate $(x<0)$ respectively.

First we consider the spanwise dependency of these functions and the $\lambda$ dependencies arising from the $Z_{n}$ basis expansion, as done by Envia (1988). We know the physical spanwise dependency of the scattered pressure jump just downstream of the trailing edge is $\sim \mathrm{e}^{\mathrm{i} k_{1} c F(\zeta)+\mathrm{i} k_{3} \zeta}$. By linearity the spanwise dependency of the pressure just upstream of the trailing edge and the velocity just downstream of the trailing edge must be identical. Therefore upon expanding the unknown Fourier transforms of the scattered pressure jump and normal velocity in the $Z_{n}$ basis we must obtain factors of $E_{n}(\lambda)$ in $A_{n}(\lambda)$ and $U_{-}^{n}(\lambda)$ corresponding to the expansion of their $\zeta$ dependencies. In (3.14) we therefore factor out $E_{n}(\lambda)$ by writing $A_{n}(\lambda)=\tilde{A}_{n}(\lambda) E_{n}(\lambda)$ and $U_{-}^{n}(\lambda)=\tilde{U}_{-}^{n}(\lambda) E_{n}(\lambda)$.

Both $\tilde{A}_{n}(\lambda)$ and $\tilde{U}_{-}^{n}(\lambda)$ could still contain unknown functions of $\lambda$ due to streamwise dependencies. To consider the streamwise dependencies we use the unsteady Kutta condition which enforces that the pressure is not singular on approach to the trailing edge $x \rightarrow 0$, therefore $\tilde{A}_{n}(\lambda) \sqrt{\lambda^{2}-w_{n}^{2}} \sim \lambda^{-1 / 2}$ and $\tilde{U}_{-}^{n}(\lambda) \sim \lambda^{-1}$ as $\lambda \rightarrow \infty$.

We therefore see both the + and - contributions to (3.15), after factorisation of $E_{n}$, decay to zero for large $\lambda$, thus the entire function they equate to must be zero. We take the + functions from $(3.15)$ to solve for $A_{n}$, yielding

$$
A_{n}(\lambda)=\frac{E_{n}(\lambda) \sqrt{-\delta-w_{n}}}{2 \mathrm{i}(\lambda+\delta) \sqrt{\lambda-w_{n}}} .
$$

This gives

$$
H(\lambda, \eta, \zeta)=\sum_{n=-\infty}^{\infty} \frac{E_{n}(\lambda) \sqrt{-\delta-w_{n}}}{2 \mathrm{i}(\lambda+\delta) \sqrt{\lambda-w_{n}}} \operatorname{sgn}(\eta) \mathrm{e}^{-|\eta| \sqrt{\lambda^{2}-w_{n}^{2}}} Z_{n}(\lambda, \zeta) .
$$

We invert the Fourier transform and apply the method of steepest descents to obtain a far-field $(r \gg 1)$ approximation as

$h(r, \theta, z) \sim \sum_{n=-\infty}^{\infty} \frac{E_{n}\left(-w_{n} \cos \theta\right) \sqrt{-\delta-w_{n}}}{2 \mathrm{i}\left(\delta-w_{n} \cos \theta\right)} Z_{n}\left(-w_{n} \cos \theta, z\right) \sin \left(\frac{\theta}{2}\right) \frac{\mathrm{e}^{\mathrm{i} w_{n} r} \mathrm{e}^{\mathrm{i} \pi / 4}}{\sqrt{\pi r}} \mathrm{e}^{\mathrm{i} w_{n} \cos \theta c F(z)}$,

where $(r, \theta, z)$ are cylindrical polar coordinates centred on $x=y=0$. We note for sufficiently large $n$ (dependent on the variables in this study) the scattered modes are cutoff i.e. $\operatorname{Im}\left(w_{n}\right)>0$, therefore in practice one only has to sum a finite number of terms in (3.18) to calculate the far-field pressure.

This completes our analytic solution for a general trailing-edge geometry, $F(z)$. 


\subsection{Limit as $c \rightarrow 0$}

We can take our general solution, (3.18), and limit $c \rightarrow 0$ to validate it with the straightedged case whose result features in Noble (1958) for $k_{3}=0$.

In this case as $c \rightarrow 0, E_{m} \rightarrow 0$ for $m \geqslant 1$, and $E_{0} \rightarrow 1$. This yields a limit, $h_{0}(r, \theta, z)=$ $\lim _{c \rightarrow 0} h(r, \theta, z)$ of

$$
h_{0}(r, \theta, z)=\frac{\sqrt{-\delta-w_{0}}}{2 \mathrm{i}\left(\delta-w_{0} \cos \theta\right)} \sin \left(\frac{\theta}{2}\right) \frac{\mathrm{e}^{\mathrm{i} w_{0} r} \mathrm{e}^{\mathrm{i} \pi / 4}}{\sqrt{\pi r}} .
$$

The corresponding limit of the Fourier transform of this solution (equivalent to (3.17) under the limit $c \rightarrow 0$ ) is

$$
H_{0}(\lambda, \eta, \zeta)=\frac{\sqrt{-\delta-w_{0}}}{2 \mathrm{i}(\lambda+\delta) \sqrt{\lambda-w_{0}}} \operatorname{sgn}(\eta) \mathrm{e}^{-|\eta| \sqrt{\lambda^{2}-w_{0}^{2}}}
$$

This is in agreement with the solution for a straight trailing edge as can be seen by comparing with the half-plate scattering problem in Noble $(1958, \S 2)$.

Thus our solution for the general trailing-edge geometry is in agreement with the straight-edged result when $c \rightarrow 0$.

\section{Results}

We now present results calculated from the analytic expression (3.18). In all cases, all propagating modes are included in the summation whilst all cutoff modes are excluded. We shall restrict to low Mach numbers $M<0.4$ as this is the range used in typical experimental tests, although higher (subsonic) Mach numbers are permitted in this analytic formulation.

\subsection{Comparison with Lyu et al. (2016)}

We have previously shown in section 3.1 that our new solution is in agreement with the straight-edged formulation. We now validate our new method for a serrated trailing-edge geometry by comparing our far-field results against those from Lyu et al. (2016) which have been compared to FEM. In particular we compare the reduction in far-field sound pressure level (SPL), denoted as $\triangle \mathrm{SPL}$, at $90^{\circ}$ above a sawtooth serrated trailing edge (compared to a straight edge) at the mid span of the serration, $z=0.5$, with $k_{3}=0$, as illustrated in Figure 5 from Lyu et al. (2016). The SPL defined in that paper is

$$
\mathrm{SPL}=20 \log _{10}|h|,
$$

where $h$ is the far-field pressure (denoted as $p_{f}$ in Lyu et al. (2016)), which from our analysis will come from (3.18) evaluated at a suitably large value of $r$ (chosen to be $r=10$ ). We also choose $k=0.7 M k_{1}$ as done so in Lyu et al. (2016) since it is claimed by Chase (1987) that the convection velocity, $U_{c}$, of the wall pressure gust is typically satisfied by $U_{c} \approx 0.7 U$, where $U$ is the uniform mean flow velocity.

We illustrate the good comparison between our new method and Lyu et al. (2016)'s method in Figure 2 for both shallow ( $c$ small) and sharp ( $c$ large) serrations. Note the results in Lyu et al. (2016) use a finite chord length to non-dimensionalise frequency, whilst we use the serration wavelength, $\lambda$. The finite chord of Lyu et al. (2016) ensures a rescattering of the trailing-edge field by the leading edge (as discussed by Roger \& Moreau (2005, 2009)) is captured by the far field results, whereas our results do not account for this. We therefore expect small variations between our results and attribute them to this additional scattering. Additionally Lyu's solution is an iterative solution, 


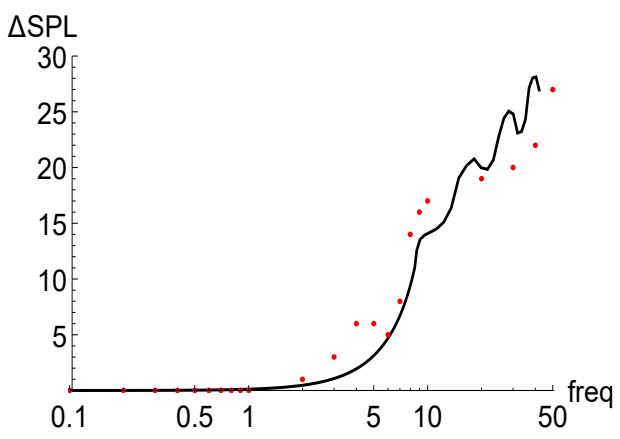

(a) $M=0.1, c=4 / 3, \lambda /($ chord length $)=0.15$ $\triangle \mathrm{SPL}(\mathrm{dB})$

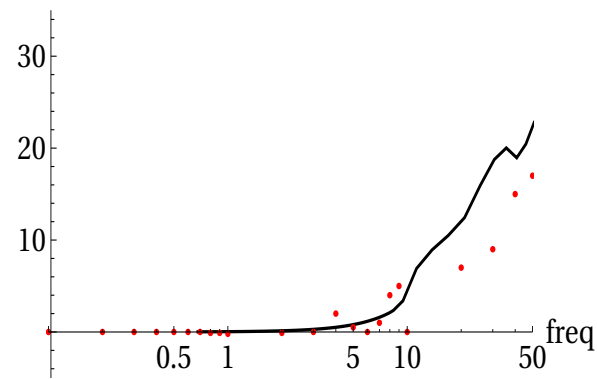

(b) $M=0.2, c=40 / 3, \lambda /($ chord length $)=$ 0.03

Figure 2: Reduction of far-field SPL $(\triangle \mathrm{SPL})$ for a serrated edge plotted against nondimensionalised frequency (freq). Results taken from Lyu et al. (2016) are plotted as red circles. Finite chord length to serration wavelength, $\lambda$, given for Lyu et al. (2016)'s results.

truncated at the second iteration. This is stated to have errors of $\sim 2 \mathrm{~dB}$ compared to the first iteration, however it is unclear what further errors exist between the second and higher iterations. By truncating the solution at the second iteration, higher-order modes are neglected in Lyu et al. (2016). The solution presented in this paper does not require truncation of higher-order modes; all propagation modes are accounted for in the far-field solution.

\subsection{SPL for different trailing-edge geometries}

In this section we present results for the far-field sound given by (4.1) for the five different test-case trailing-edge geometries shown in Figure 1. We label each geometry (a)-(e) as $\mathrm{S}, \mathrm{SV}, \mathrm{SU}, \mathrm{CP}, \mathrm{SQ}$ respectively.

Specifically the geometries are given by

$$
\begin{aligned}
& \text { sawtooth } \quad F_{S}(z)= \begin{cases}z, & z \in\left[0, \frac{1}{4}\right) \\
\frac{1}{2}-z, & z \in\left(\frac{1}{4}, \frac{3}{4}\right), \\
z-1, & z \in\left(\frac{3}{4}, 1\right]\end{cases} \\
& \text { slitted v-root } \quad F_{S V}(z)= \begin{cases}\frac{2}{3} z, & z \in\left[0, \frac{1}{4}\right) \\
\frac{2}{3}\left(\frac{1}{2}-z\right), & z \in\left(\frac{1}{4}, \frac{7}{10}\right) \\
\frac{8}{3}-4 z & z \in\left(\frac{7}{10}, \frac{3}{4}\right), \\
-\frac{10}{3}+4 z & z \in\left(\frac{3}{4}, \frac{4}{5}\right) \\
\frac{2}{3}(z-1), & z \in\left(\frac{4}{5}, 1\right]\end{cases} \\
& \text { slitted u-root } \quad F_{S U}(z)= \begin{cases}\frac{2}{3} z, & z \in\left[0, \frac{1}{4}\right) \\
\frac{2}{3}\left(\frac{1}{2}-z\right), & z \in\left(\frac{1}{4}, \frac{7}{10}\right) \\
-\frac{1}{3} & z \in\left(\frac{7}{10}, \frac{4}{5}\right) \\
\frac{2}{3}(z-1), & z \in\left(\frac{4}{5}, 1\right]\end{cases}
\end{aligned}
$$




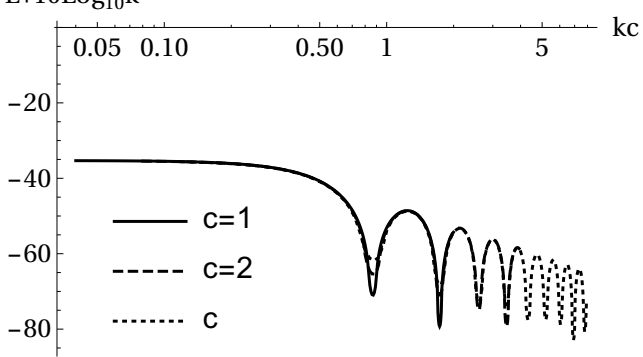

(a) Serration (a)

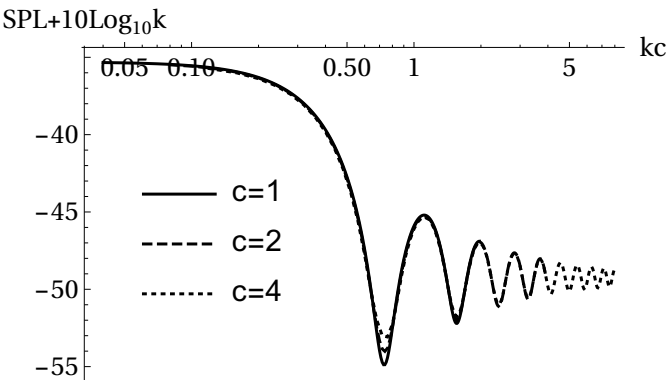

(b) Chopped peak (d)

Figure 3: Scaled pressure spectra with scaled frequency for two different trailing-edge geometries, at $M=0.1$, with $k_{3}=0$.

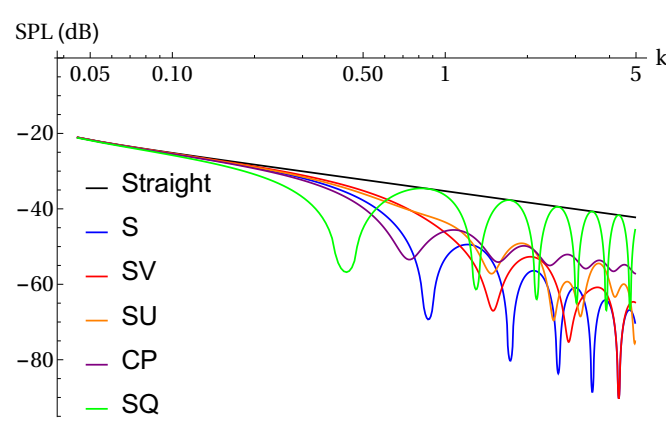

(a) $M=0.1$.

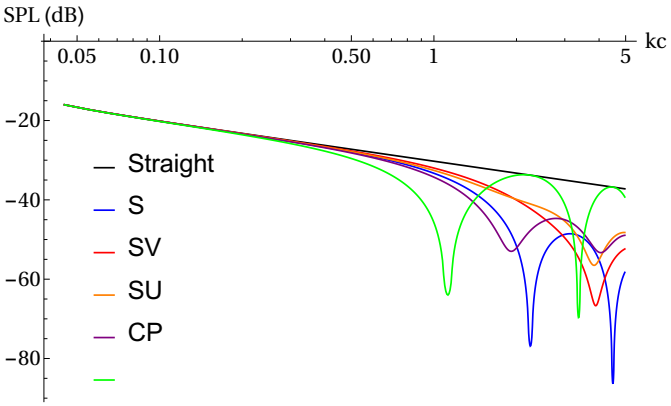

(b) $M=0.3$.

Figure 4: Pressure spectra for trailing-edge geometries (a) to (e), with $k_{3}=0$. Colour online

$$
\begin{aligned}
\text { chopped peak } & F_{C P}(z)=\frac{5}{4} \begin{cases}z, & z \in\left[0, \frac{3}{20}\right) \\
\frac{3}{20} & z \in\left(\frac{3}{20}, \frac{7}{20}\right) \\
\frac{1}{2}-z & z \in\left(\frac{7}{20}, \frac{3}{4}\right) \\
z-1, & z \in\left(\frac{3}{4}, 1\right],\end{cases} \\
\text { square wave } & F_{S Q}(z)=\frac{1}{4} \begin{cases}1, & z \in\left[0, \frac{1}{4}\right) \\
-1, & z \in\left(\frac{1}{4}, \frac{3}{4}\right) . \\
1, & z \in\left(\frac{3}{4}, 1\right]\end{cases}
\end{aligned}
$$

First we note a collapse of the results through a suitable scaling of $k$ and SPL as shown in Figure 3, therefore we shall consider results from here as functions of scaled frequency $k c$.

We plot SPL against scaled frequency for the five different trailing edge geometries in Figure 4 for different flow speeds. Whilst this does not indicate the reduction of noise for a realistic flow over a serrated trailing edge (as we have restricted to $k_{3}=0$ only) it provides us a foundation on which to understand the key influential parameters in the analytic solution and how they affect the far-field noise.

It is clear from Figure 4 that different trailing-edge geometries are best at reducing scattered noise at different mid-range frequencies, however at high frequencies the sawtooth (S) and serrated v-root (SV) seem preferential over the other geometries. This 
variation in optimal SPL reduction occurs because every SPL for a non-straight edge exhibits oscillatory behaviour as $k c$ increases. This indicates an interference effect in the far-field, in particular where there are minima of the spectra there is a strong destructive interference between acoustic fields scattered at the tip and at the root of each geometry. The chopped peak (CP) exhibits the weakest oscillation (smallest amplitude variation in the oscillations) indicating there is weaker tip and root interference, whilst the square wave (SQ) has the greatest oscillation indicating a strong tip and root interference. The interference is clearly dependent on the flow speed, $M$, and the specific trailing-edge geometry as all oscillations vary as we change these parameters.

The modal expansion coefficients, $E_{n}\left(-w_{n} \cos \theta\right)$ found in (3.18), are key to understanding the tip and root interference. We illustrate this by considering $E_{0}\left(-w_{0} \cos \theta\right)$ (the zeroth modal coefficient) for each geometry at $\theta=90^{\circ}$ and $k_{3}=0$. These coefficients are calculated from (3.13) and given by

$$
\begin{aligned}
E_{0}^{S}(s) & =\frac{1}{s} \sin (s) \\
E_{0}^{S V}(s) & =\frac{\mathrm{ie}^{-\mathrm{i} s / 3}}{2 s}\left(1+5 \mathrm{e}^{\mathrm{i} s / 5}-6 \mathrm{e}^{\mathrm{i} s / 2}\right), \\
E_{0}^{S U}(s) & =\frac{\mathrm{e}^{-\mathrm{i} s / 3}}{10 s}\left(s-30 \mathrm{ie}^{\mathrm{i} s / 5}\left(\mathrm{e}^{3 \mathrm{i} s / 10}-1\right)\right), \\
E_{0}^{C P}(s) & =\frac{\mathrm{e}^{-5 \mathrm{i} s / 16}}{5 s}\left(s \mathrm{e}^{\mathrm{i} s / 2}-8 \mathrm{i}\left(\mathrm{e}^{\mathrm{i} s / 2}-1\right)\right), \\
E_{0}^{S Q}(s) & =\cos \left(\frac{s}{4}\right) .
\end{aligned}
$$

where the superscript denotes which geometry we are considering, and $s=\delta c / \beta$.

In Figure 5 we plot $20 \log _{10}\left|E_{0}(s)\right|$ for each of the edge geometries and compare to Figure $4 \mathrm{a}$. We see the oscillations given by these $E_{0}$ functions very closely match the oscillations of the full SPL. We also see the relative magnitudes of the $E_{0}$ functions match the full SPL. The relative behaviour of the SPL is therefore dominated by the zeroth modal expansion term, $E_{0}$. Higher order modes have an effect once they are cut on; this requires $k \beta^{-1}-2 n \pi>0$ for the nth mode to be cut on.

The $E_{0}$ modal coefficients therefore provide us with a very simple formulation for the expected interference of the root and tip, as these coefficients can be calculated from (3.13) without any need for the Wiener-Hopf method. The coefficients also indicate, when $k_{3}=0$, why for large $k c$ values the sawtooth (S) and serrated v-root (SV) are better at reducing noise than the other geometries. If we consider the large $s$ (corresponding to large $k c$ ) behaviour of (4.3), we see that the sawtooth and serrated v-root coefficients behave as $O\left(s^{-1}\right)$, whilst the remaining geometries behave as $O(1)$. Therefore we expect for increasingly sharp serrations, the sawtooth and serrated v-root reduce noise compared to the straight edge as $\sim \log (k c)$, however the other geometries (the serrated u-root, chopped peak, and square wave) will tend to a constant noise reduction.

To ensure this conclusion still holds when higher modes are cuton, we consider the higher modal coefficients $E_{n}$. For simplicity we illustrate the behaviour with the sawtooth coefficients, $E_{n}^{S}\left(-w_{n} \cos \theta\right)$, and the square wave coefficients, $E_{n}^{S Q}\left(-w_{n} \cos \theta\right)$, as these are indicative of the two different cases which can occur. We again evaluate these at $\theta=90^{\circ}$ and $k_{3}=0$, therefore $E_{n}$ for these two geometries are given by

$$
E_{n}^{S}(s)=\frac{-4 s \mathrm{e}^{-\mathrm{i} n \pi}}{4 n^{2} \pi^{2}-s^{2}} \sin \left(\frac{1}{4}(2 n \pi+s)\right) .
$$




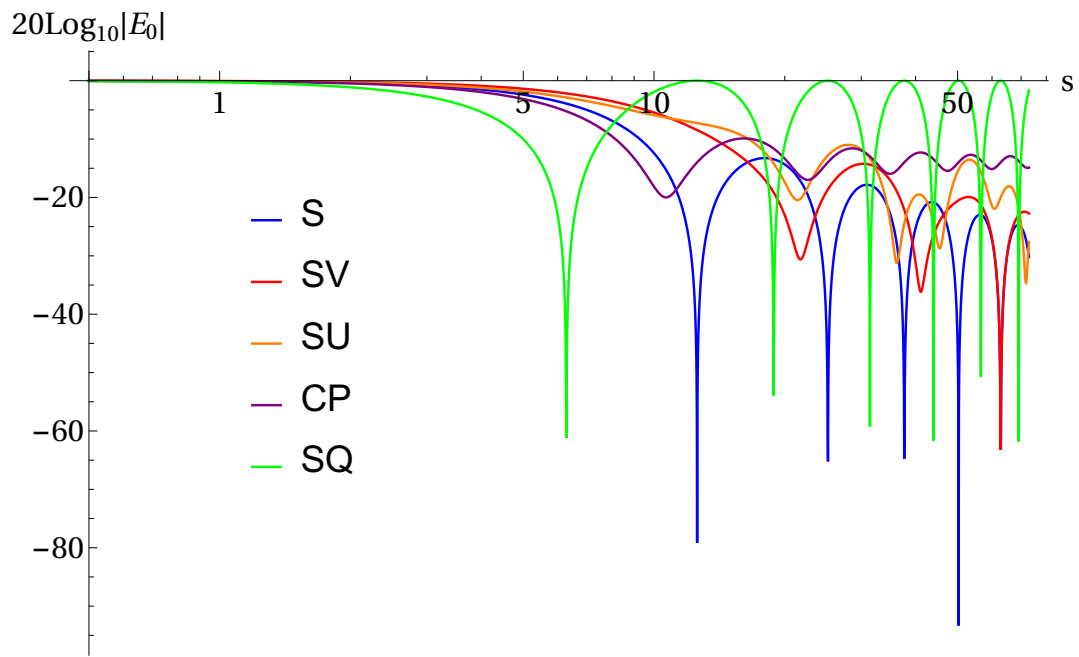

Figure 5: $20 \log _{10}\left|E_{0}(s)\right|$, for $k_{3}=0$, for trailing-edge geometries (a) to (e). Colour online

$$
E_{n}^{S Q}(s)=\left\{\begin{array}{l}
\frac{\mathrm{ie}^{\mathrm{i}(n-1) \pi / 2}}{n \pi} \sin \left(\frac{s}{4}\right) \quad n \text { odd } \\
0 \quad n \text { even }
\end{array}\right.
$$

For large $s$, the $n$th mode coefficients behave similarly to the zeroth mode coefficients, with the exception that for the mode $n \approx s / 2 \pi, E_{n}^{S}(s) \sim-1 / 2$. Our conclusion therefore, of the sawtooth and serrated v-root experiencing a reduction of noise as $\sim \log (k c)$ for increasing $k c$, still holds, and we predict these geometries are preferential for large $k c$ values even when higher modes contribute. The emergence of a large $n$ mode whose coefficient does not decay with $k c$ suggests there is a redistribution of the acoustic energy from lower cuton modes to these higher cutoff modes as the serration is sharpened (as $c$ increases).

\subsection{Far-Field Directivity}

Here we consider the effect of varying $\theta$ on the far-field SPL by plotting the directivity patterns (defined as SPL plotted as a function of observer angle $\theta$ ). It is known that SPL varies as observer angle $\theta$ varies, more so with higher frequency interactions (Lyu et al. 2016).

In Figure 6 we show the mid $\operatorname{span}(z=0.5)$ directivity patterns at $r=10$ for a variety of values of $k c$. To enable all values to be easily visible, we add a constant reference value of $20 \log _{10} 10^{6}$ to each SPL. We see the directivities overall follow the same trends as Figure 4 despite having variations with observer angle as $k c$ increases. The sawtooth (S), v-root (SV), and square wave (SQ) all exhibit strong oscillations with varying observer angle (again illustrating their strong tip and root interference), therefore we must be careful to include a range of observer angles when determining the overall effectiveness of these edges. For example measuring only at $\theta=90^{\circ}$ could over-predict the noise reduction if this coincides with a minima in the directivity oscillations. These oscillations can also cause different edges to appear optimal at a given $k c$ value and given observer angle, when overall they are not; Figure $6 \mathrm{~d}$ shows the sawtooth $(\mathrm{S})$ generating more noise at $\theta=90^{\circ}$ than the v-root (SV), however further towards the downstream direction, $\theta=130^{\circ}$, the sawtooth generates significantly less noise than the v-root.

Over almost all observer angles there is a reduction of far-field noise, as observed by Sanjose et al. (2014). In the wake direction $(\theta=0)$ Sanjose et al. (2014) observe a slight 


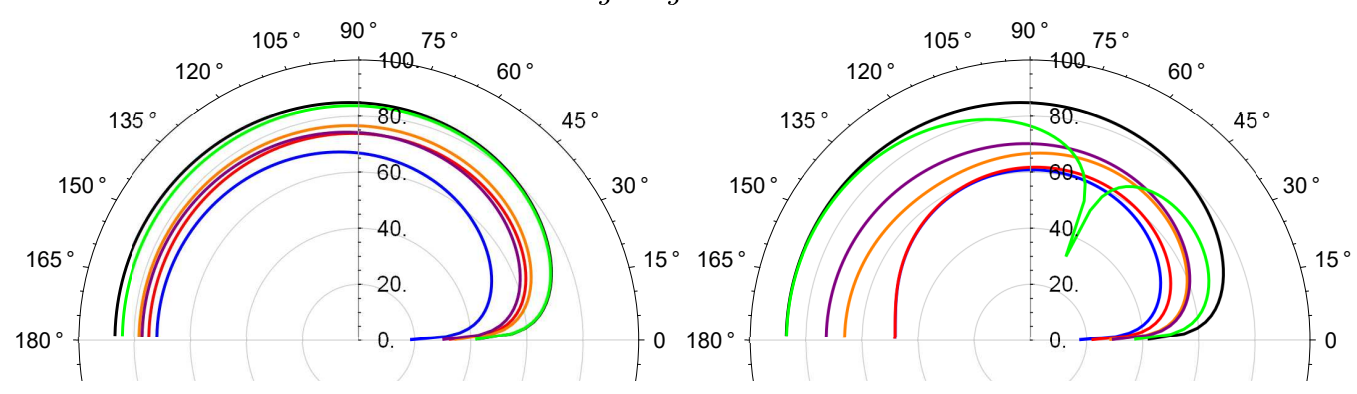

(a) $k c=1$.

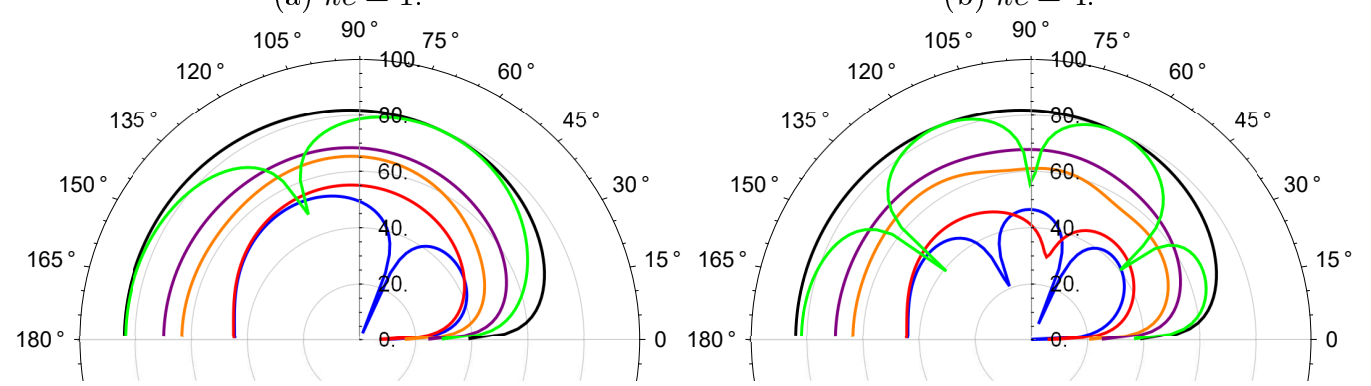

(c) $k c=8$.

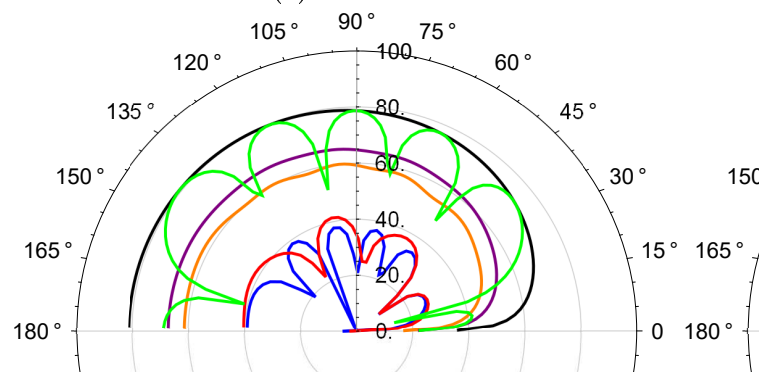

(e) $k c=32$. (d) $k c=16$.

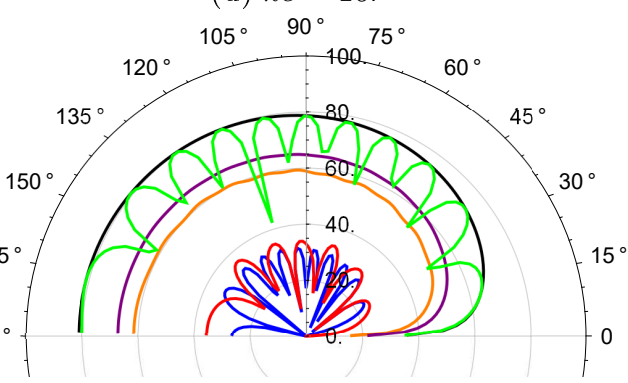

(f) $k c=64$.

Figure 6: Far-field SPL directivities, $20 \log _{10}\left(10^{6}|h(10, \theta, 0.5)|\right)$, for $M=0.1, k_{3}=0$. Colours match Figure 4; straight - black, S - blue, SV - red, SU - orange, CP - purple, $\mathrm{SQ}$ - green.

noise increase, which we do not see here. This may be caused by the vortex shedding (more precisely the reduction of vortex shedding) by the serrated edge. As our simple analytical model assumes a vortex sheet for the wake, we cannot assess any effects of altered vortex shedding, and therefore do not recreate this result.

\subsection{Far-field sound spectra}

In the previous two sections we have focused on $k_{3}=0$, however to have a prediction of a realistic level of noise scattered by a trailing edge we must include a range of $k_{3}$ values. To do so, we define the far-field power spectral density (PSD) as

$$
\mathrm{PSD}=10 \log _{10}\left(\int_{-\infty}^{\infty}|h(r, \theta, z)|^{2} \Pi\left(\omega, k_{3}\right) d k_{3}\right),
$$


where

$$
\Pi\left(\omega, k_{3}\right) \sim \frac{\left(\omega / U_{c}\right)^{2}}{\left(\left(\left(\omega / U_{c}\right)^{2}+k_{3}\right)^{2}+L^{2}\right)^{2}}
$$

is the leading-order approximation from Howe (1991a) for the wavenumber spectral density in a turbulent boundary layer proposed by Chase (1987) (and that used by Lyu et al. (2016)) under the assumption $k_{1} \approx \omega / U_{c}$. Overall multiplicative constants which are independent of the edge geometry have been removed from this definition for clarity. The parameter $L$ is defined as $L=\chi / \delta_{B L}$, where $\chi \sim 1.33$ is provided by Chase (1987), and $\delta_{B L}$ is the (non-dimensional) boundary layer thickness. We too use the value $\chi=1.33$ in this paper in accordance with previous analytic models (Lyu et al. 2016; Howe 1991b,a), although note in different experimental and numerical setups this parameter (and indeed the assumption $U_{c} \approx 0.7 U$ can vary).

In Figure 7 we present the far-field PSD for each of the trailing-edge geometries at $\theta=90^{\circ}$. We see the oscillatory behaviour of the SPL (Figure 4 ) is weakened but still present, and we see once again that sharper serrations (larger values of $c$ ) generally result in a larger noise reduction at a given frequency for each geometry. What differs in the PSD compared to the SPL however is the noise reduction for high frequencies; we see when including a spectrum of $k_{3}$ values, the PSD reduction for any serrated edge verses a straight edge can be decreased at high frequencies. There is therefore additional noise created by the serrated edges by $k_{3} \neq 0$ components which is not created by the straight edge, particularly at high frequencies. This can be seen from the modes scattered by the serrated edge, (3.9); for $k_{3} \sim 2 n \pi$ the nth mode will always be cuton. This mode does not exist for a straight edge, whose only scattered frequency is $w_{0}^{2}=(k / \beta)^{2}-k_{3}^{2}$. The redistribution of energy from lower cuton modes to higher cutoff modes discussed for $k_{3}=0$ now becomes more complicated as the higher modes that energy is redistributed to for increasing frequency or tip to root height (increasing $k c$ ) become cuton when including sufficiently large $k_{3}$ spectral values.

\subsection{Comparison with experimental measurements}

In this section we compare the analytically predicted far-field reduction of PSD with previous analytical, numerical and experimental measurements, for a sawtooth (S) trailing edge verses a straight trailing edge.

In the analytic model in this paper the mean flow is always parallel to the serrated edge, and we use a simple wall turbulence model described by the wavenumber spectrum (4.7), as assumed by Lyu et al. (2016); Howe (1991b). It is known that total aerofoil angle of attack (Gruber 2012) and relative misalignment angle of the serrations to the chord (Leon et al. 2017) can have a significant effect on the predicted far-field noise (up to $\sim 4 \mathrm{~dB}$ and $\sim 10 \mathrm{~dB}$ respectively for large angles). In Leon et al. (2017) these effects are attributed to the distorted near-surface mean flow and the formation of streamwise turbulent structures. The simple wavenumber spectrum used analytically would likely be unsuitable for describing the turbulence over a serrated trailing-edge aerofoil at significant angle of attack or with misaligned serrations due to their effect on the turbulent structures. Additionally, the effect of vortex shedding, which will occur particularly prominently for aerofoils with serrations cut into the main body (thus having regions along the trailing edge with blunt sections) is not accounted for in the analytical model, although this will produce a discrepancy of tonal rather than broadband noise.

We compare our new analytic solution against a variety of experimental, numerical, and previous analytical models in Figure 8. Compared to Howe's analytical model, the current analytic prediction for noise reduction is reduced, as would be expected given 


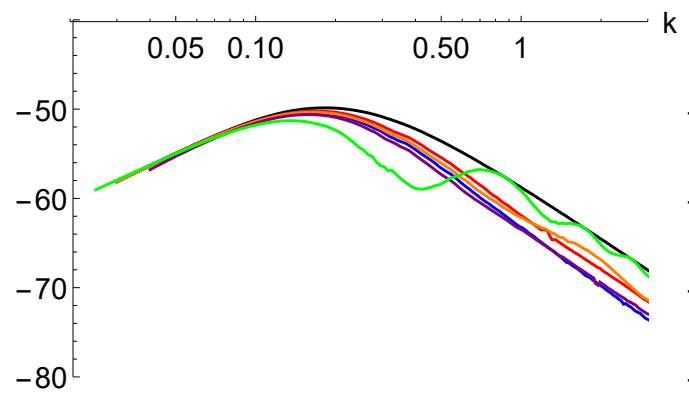

(a) $c=1$.

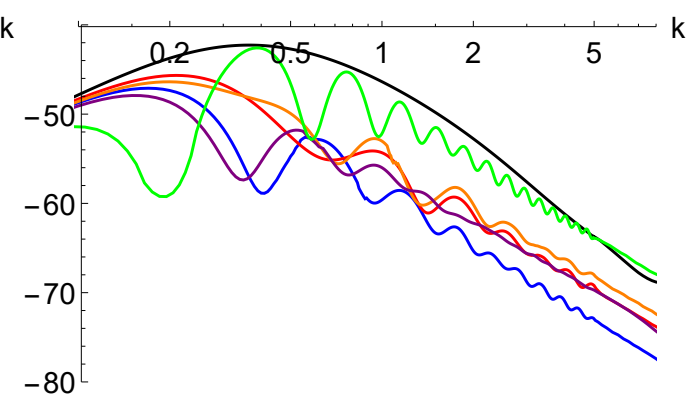

(b) $c=4$.

Figure 7: Far-field PSD, for $M=0.1, L=1.33 / 0.5$. Colours match Figure 4; straight black, S - blue, SV - red, SU - orange, CP - purple, SQ - green.

the known overprediction of Howe's results (Lyu et al. 2016; Winkler et al. 2010). We see in general good agreement with Lyu's results (Lyu et al. 2016), and mention again, on inclusion of a $k_{3}$ wavenumber spectrum, the higher-order modes (which are neglected by Lyu) become important as modes with $k_{3} \sim 2 n \pi, n \in \mathbb{Z}$, always propagate to the far-field.

The poor agreement with the experimental results of Gruber (2012) is attributed to the high effective angle of attack at the trailing edge (due to both an angle of attack of the flow, and a cambered aerofoil).

The numerical results of Jones \& Sandberg (2012) found through DNS, show a similar trend to Howe's predictions, in that there is no drop-off of noise reduction at higher frequencies. This is mirrored in the experimental results of Chong et al. (2013), and indeed in our new analytical model to the frequencies investigated in Figure 8 . As the numerical and experimental results are at a $\sim 5^{\circ}$ angle of attack, we can expect up to $\sim 4 \mathrm{~dB}$ difference in noise reduction (Gruber 2012) verses the flat-plate case investigated analytically at high frequencies, which is seen here.

We also mention that at low frequencies, the steepest descent approximation made prior to (3.18) is no longer valid, therefore we expect discrepancies between our current analytic results and all other results for $f \lesssim 400 \mathrm{~Hz}$.

Overall it is clear from the results present in Figure 8 there is great variation in the prediction of noise reduction for serrated trailing edges, due to a variety of aerofoil and flow parameters. However, overall the new analytic results found in this paper are an improvement over Howe's predictions and can form a basis for developing more involved analytical models which could take into account angle of attack or geometry. Furthermore, the new solution presented in this paper can account for all propagating modes and does not require an iterative numerical procedure to produce results, which is an improvement over the method presented by Lyu et al. (2016).

\section{Conclusions}

In this paper we have found an analytic solution for the far-field noise generated by the turbulent boundary layer scattering off of a trailing edge which is permitted some periodic spanwise variation in geometry. The analytic solution, obtained via the use of a non-orthogonal coordinate transformation, separation of variables, and the Wiener-Hopf technique, leads to a closed-form prediction of the far-field noise. The solution limits to the known straight-edge solution as the serration height is sent to zero, and compares 
16

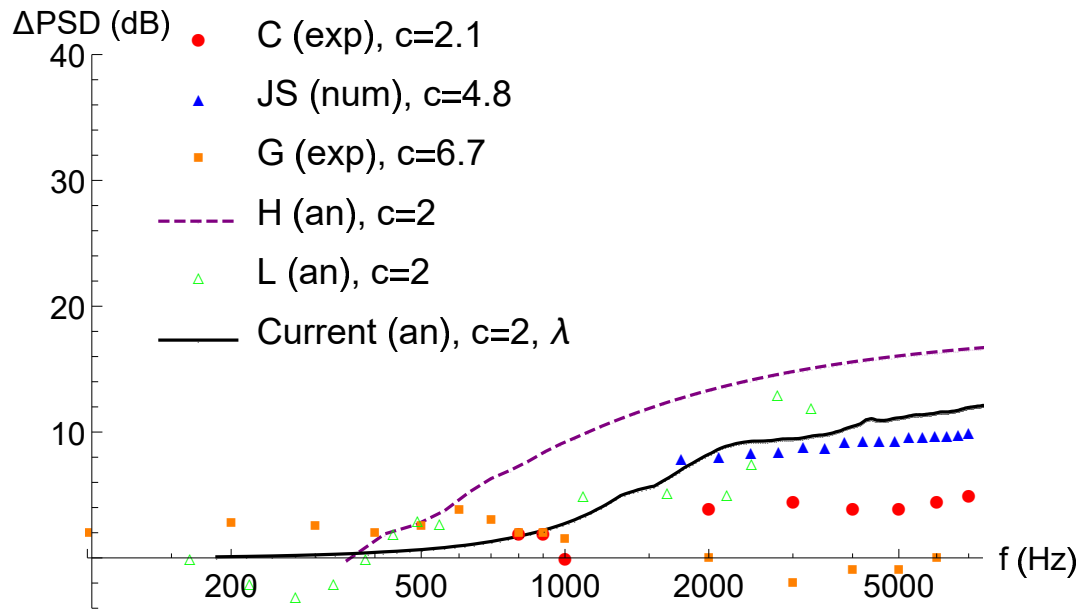

(a) Analytic solutions with $c=2, M=0.1, \lambda=19 \mathrm{~mm}$ (as used by Chong et al. (2013, Fig. 9)).

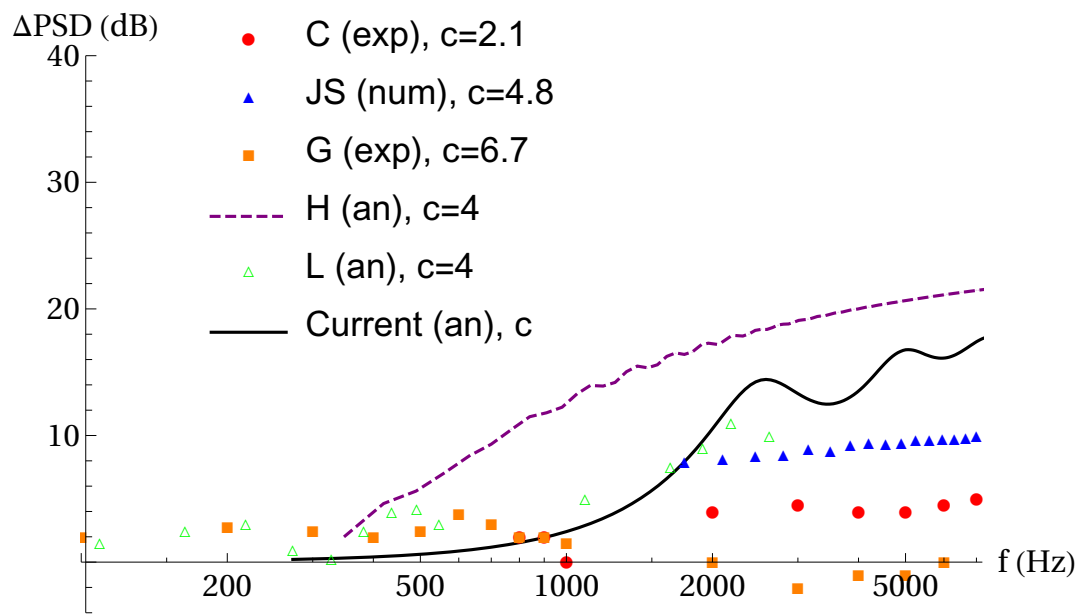

(b) Analytic solution with $c=4, M=0.1$, and $\lambda=9 \mathrm{~mm}$ (as used by Gruber (2012, Fig. 4.4)).

Figure 8: PSD noise reduction in $\mathrm{dB}$ for a serrated edge versus a straight edge as a function of frequency $(\mathrm{Hz})$. Experimental results taken from Chong et al. (2013, Fig 9) (red dots) for a NACA 0012 aerofoil at $4.2^{\circ}$ angle of attack with $U_{\infty}=51 \mathrm{~m} / \mathrm{s}$. Numerical results (blue triangles) taken from (Jones \& Sandberg 2012, Fig 6) for NACA 0012 aerofoil at $5^{\circ}$ angle of attack, with $M=0.4$. Experimental results taken from Gruber (2012, Fig 4.4) (orange squares) for a NACA65(12)-10 aerofoil at $5^{\circ}$ angle of attack, with $U_{\infty}=40 \mathrm{~m} / \mathrm{s}$. Previous analytical results are from Howe (1991b, Eq. 18) (purple dashed line, with $h / \delta=8$ as defined in Howe's solution) and Lyu et al. (2016, Fig 6c) (green triangles) both taken directly above the trailing edge at $\theta=90^{\circ}$. Current analytic results (black line) calculated from (4.6) use $L=1.33 / 0.5$, taken at $\theta=90^{\circ}$.

well with the previous semi-analytic results of Lyu et al. (2016) for sawtooth serrations both with small and large tip to root ratios. With this simple analytic expression for the far-field pressure, we have investigated the effects of different trailing-edge geometries on 
the overall noise levels, and determined mechanisms which allow different geometries to reduce the noise levels differently.

There are two key mechanisms of noise reduction. First a destructive interference of tip and root fields, which dominates in the low and mid-frequency ranges, and is most beneficial for the square wave (SQ) geometry. Second, a redistribution of acoustic energy from low propagating modes to higher cutoff modes, which dominates in the highfrequency range, and is only fully effective for geometries without any flat sections, such as the serration (S) and slitted v-root serration (SV). When considering a spectrum of $k_{3}$ values this second feature is impeded; higher modes become cuton for $k_{3} \sim 2 n \pi$, and therefore do still propagate to the far field.

We have seen that very large noise reductions can be seen at specific observer angles in the far field, but due to modulated directivity patterns if one considers the whole range of observer angles the noise reduction is lessened (but still effective). One must therefore be careful if conducting experiments to measure a sufficiently large range of observer angles to avoid over-predicting the noise reduction possible for a given trailing-edge design.

It is known that a trailing-edge spanwise variation can affect the turbulent structures at the trailing edge (Avallone et al. 2016), therefore it is unclear if using the simplistic wavenumber spectrum from Chase (1987) is sufficiently realistic to predict true noise reductions from serrated trailing edges. It may be valuable to develop a new wavenumber spectrum that realistically matches the wall pressures measured experimentally at serrated trailing edges. This analytic method would still work with any such appropriate spectrum. It is also known that serrated trailing edges can significantly affect the structure of the turbulent wake (Jones \& Sandberg 2012; Sanjose et al. 2014) and in particular the structure of the shed vortices. Since this analytic model uses a vortex sheet it does not account for any alterations to the wake due to the existence of the serrations.

Whilst we have only considered five test case geometries in this paper, the method permits any single-valued periodic geometry. This work can therefore be used to predict the optimal version of any trailing-edge design, such as the optimal slit width for a slitted v-root serration. This, of course, should be balanced with minimising the aerodynamic impact of reducing the surface area at the trailing edge, and should be a subject of future research.

\section{Acknowledgements}

The work in this paper was supported by EPSRC Early Career Fellowship EP/P015980/1.

\section{REFERENCES}

Aмiet, R.K. 1976 Noise due to turbulent flow past a trailing edge . Journal of Sound and Vibration 47 387-393.

Avallone, F., Probsting, S. and Ragni, D. 2016 Three-dimensional flow field over a trailingedge serration and implications on broadband noise. Physics of Fluids 28117101.

Avallone, F., van der Velden, W. C. P. and Ragni, D. 2017 Benefits of curved serrations on broadband trailing-edge noise reduction. Journal of Sound and Vibration 400 167-177.

Ayton, L. J. \& Kim, J. W. 2018 An analytic solution for the noise generated by gust-aerofoil interaction for plates with serrated leading edges. arXiv:1805.05118.

Azarpeyvand, M., Gruber, M. \& Joseph, P. 2013 An analytical investigation of trailing edge noise reduction using novel serrations. 19th AIAA/CEAS Aeroacoustics Conference, AIAA 2013-2009.

Cavalieri, A. V. G. Wolf, W. R. \& Jaworski, J. W. 2016 Numerical solution of acoustic scattering by finite perforated elastic plates. P. Roy. Soc. A 427, 20150767.

Chase, D. M. 1987 The character of the turbulent wall pressure spectrum at subconvective 
wavenumbers and a suggested comprehensive model. Journal of Sound and Vibration 112, $125-147$.

Chong, T. P. \& Vathylakis, A. 2015 On the aeroacoustic and flow structures developed on a flat plate with a serrated sawtooth trailing edge. Journal of Sound and Vibration 354, 65-90.

Chong, T. P., Vathylakis, A., Joseph, P. F. \& Gurber, M. 2016 Self-Noise Produced by an Airfoil with Nonflat Plate Trailing-Edge Serrations. AIAA Journal 51, 2665-2677.

Clark, i.A, Devenport, W., Jaworski, J.W., Daly, C. A., Peake, N., \& Glegg, S. 2014 The noise generating and suppressing characteristics of bio-inspired rough surfaces. 20th AIAA/CEAS Aeroacoustics 2911.

Clark, I.A, Daly, C. A., Devenport, W., Alexander, W. N., Peake, N., Jaworski, J.W., \& GleGG, S. 2016 Bio-inspired canopies for the reduction of roughness noise. Journal of Sound and Vibration, 385 33-54.

Crighton, D.G., Dowling, A.P., Williams, J.F., Heckl, M. \& Leppington, F.G. 1996 Modern Methods in Analytical Acoustics. Springer.

Dassen, T., Parchen, R., Bruggeman, J. and Hagg, F. 1996 Results of a wind tunnel study on the reduction of airfoil self-noise by the application of serrated blade trailing edges. European Union Wind Energy Conference and Exhibition, Gothenburg. NLR TP 96350.

EnviA, E. 1988 Influence of Vane Sweep on Rotor-Stator Interaction Noise.. PhD thesis, University of Arizona.

European Commission 2011 Flightpath 2050: Europe's Vision for Aviation. Report of the High Level Group on Aviation Research, Publications Office of the European Union, Luxembourg.

Graham, R. R. 1934 The silent flight of owls. Journal of the Royal Aeronautical Society 38, 837-843.

Gruber, M. 2012 Airfoil noise reduction by edge treatments. Ph.D Thesis, University of Southampton.

Herr, M. 2006 Experimental study on noise reduction through trailing-edge brushes. Notes on Numerical Fluid Mechanics 92, 365-372.

Howe, M. S. 1991 Aerodynamic noise of a serrated trailing edge. Journal of Fluids and Structures 5, 33-45.

Howe, M. S. 1991 Noise produced by a sawtooth trailing edge. Journal of the Acoustical Society of America 90, 482-487.

Howe, M. S. 1998 Acoustics of Fluid-Structure Interactions. Cambridge University Press.

Howe, M. S. 1978 A Review of the Theory of Trailing Edge Noise. NASA Technical Report, 3021.

HuAng, X. 2017 Theoretical model of acoustic scattering from a flat plate with serrations. Journal of Fluid Mechanics 819, 228-257.

JAWORSki, J.W. \& PEAKe, N. 2013 Aerodynamic noise from a poroelastic edge with implications for the silent flight of owls. Journal of Fluid Mechanics 723, 456-479.

Jones, L. \& SAndBerg, R. D. 2010 Numerical investigation of airfoil self-noise reduction by addition of trailing edge serrations. 16th AIAA/CEAS Aeroacoustics Conference, AIAA 2010-3703.

Jones, L. \& Sandberg, R. D. 2012 Acoustic and hydrodynamic analysis of the flow around an aerofoil with trailing-edge serrations. Journal of Fluid Mechanics 706, 295-322.

Karimi, M., Croaker, P., Kinns, R. And Kessissoglou, N. 2017 Effect of a serrated trailing edge on sound radiation from nearby quadrupoles. Journal of the Acoustical Society of America 141, 2997.

Koegler, K. U., Herr, S. \& Fisher, M. 2009 Wind turbine blades with trailing edge serrations. US Patent App. 11/857,844.

Leon, C. A., Merino-Martínez, R., Ragni, D., Avallone, F., Scarano, F., Probsting, S., Snellen, M., Simons, D. G. and Madsen, J. 2017 Effect of trailing edge serrationflow misalignment on airfoil noise emissions. Journal of Sound and Vibration 406, 19-33.

Lyu, B., Azarpeyvand, M. \& Sinayoko, S. 2016 Prediction of noise from serrated trailing edges. Journal of Fluid Mechanics 793, 556-588.

Moreau, D. J. \& Doolan, C. J. 2013 Noise-Reduction Mechanism of a Flat-Plate Serrated Trailing Edge. AIAA Journal 51,2513-2522 
Noble, B. 1958 Methods Based on the Wiener-Hopf Technique for the Solution of Partial Differential Equations. Pergamon Press.

Oerlemans, S. 2016 Reduction of wind turbine noise using blade trailing edge devices. 22nd AIAA/CEAS Aeroacoustics AIAA 2016-3018.

Oerlemans, S. \& Olsen, A. S. 2014 A wind turbine blade with a noise reducing device. US Patent App. 14/427,326.

Roger, M., Schram, C. \& De Santana, L. 2013 Reduction of airfoil turbulence-impingement noise by means of leading-edge serrations and/or porous material, 19th AIAA/CEAS Aeroacoustics, AIAA 2013-2108.

Roger, M. \& Moreau, S. 2005 Back-scattering correction and further extensions of Amiet's trailing-edge noise model. Part 1: theory, Journal of Sound and Vibration, 286, 477-506.

Roger, M. \& Moreau, S. 2009 Backscattering correction and further extensions of Amiet's trailing-edge noise model. Part II: application, Journal of Sound and Vibration, 323, 397425 .

Sanjose, M., Meon, C., Masson, V., \& Moreau S. 2014 Direct numerical simulation of acoustic reduction using serrated trailing-edge on an isolated airfoil, 20th $A I A A / C E A S$ Aeroacoustics 2324.

Schlanderer, S. C. \& Sandberg, R. D. 2016 DNS of Noise Radiation from a Turbulent Flow Convecting over an Elastic Trailing-Edge. 22nd AIAA/CEAS Aeroacoustics 2836.

Winkler, J. Moreau, S. \& Carolus, T. 2010 Airfoil trailing edge noise prediction from large-eddy simulation: influence of grid resolution and noise model formulation. 16th AIAA/CEAS Aeroacoustics $\mathbf{3 7 0 4}$.

Wolf, A., Lutz, T., Würz, W., Krämer, E., Stalnov, O., \& Seifert, A. 2014 Trailing edge noise reduction of wind turbine blades by active flow control. Wind Energy 18, 909923.

van der Velden, W. C., Avallone, F. \& Ragni, D. 2017 Numerical analysis of noise reduction mechanisms of serrated trailing edges under zero lift condition. 23rd AIAA/CEAS Aeroacoustics Conference, AIAA, 2017-4173. 